\title{
Effect of curcumin on aging retinal pigment epithelial cells
}

This article was published in the following Dove Press journal:

Drug Design, Development and Therapy

25 September 2015

Number of times this article has been viewed

\author{
Wei Zhu',* \\ Yan $\mathrm{Wu}^{2, *}$ \\ Yi-Fang Meng' \\ Jin-Yu Wang' \\ Ming $X u^{\prime}$ \\ Jian-Jun Tao' \\ Jiong Lu'
}

'Department of Ophthalmology, Changshu No 2 People's Hospital, Changshu, ${ }^{2}$ Department of Ophthalmology, The First People's Hospital of Kunshan Affiliated with Jiangsu University, Suzhou, People's Republic of China

*These authors contributed equally to this work
Correspondence: Jiong Lu

Department of Ophthalmology, Changshu No 2 People's Hospital, Haiyu South Road 68, Changshu, Jiangsu 215000,

People's Republic of China

Email cslujiong@163.com
Abstract: Age-related macular degeneration (AMD) is now one of the leading causes of blindness in the elderly population. The antioxidative effects of curcumin on aging retinal pigment epithelial (RPE) cells are still unclear. We conducted an in vitro study to investigate the effects of curcumin on aging RPE cells. A pulsed $\mathrm{H}_{2} \mathrm{O}_{2}$ exposure aging model was adopted. Aging RPE cells were treated with curcumin $20 \mu \mathrm{M}, 40 \mu \mathrm{M}$, and $80 \mu \mathrm{M}$. Apoptosis of RPE cells was analyzed by flow cytometry. The intracellular reactive oxygen species concentration was detected using a specific probe and apoptosis-associated proteins were detected by Western blot. Expression of oxidative biomarkers, including superoxide dismutase, maleic dialdehyde, and glutathione, was detected commercially available assay kits. Compared with normal cells, lower cell viability, higher apoptosis rates, and more severe oxidation status were identified in the aging RPE cell model. Curcumin improved cell viability and decreased apoptosis and oxidative stress. Further, curcumin had a significant influence on expression of apoptosis-associated proteins and oxidative stress biomarkers. In conclusion, treatment with curcumin was able to regulate proliferation, oxidative stress, and apoptosis in aging RPE cells. Accordingly, application of curcumin may be a novel strategy to protect against age-related change in AMD.

Keywords: curcumin, retinal pigment epithelium, apoptosis, age-related macular degeneration

\section{Introduction}

Age-related macular degeneration (AMD) is one of the leading causes of blindness in the elderly population worldwide, ${ }^{1}$ and treatments available for the disease remain limited. Photodynamic therapy and anti-vascular endothelial growth factor agents can improve the condition in patients with neovascular AMD, but there are still no curative methods available. ${ }^{2}$ Thus, the only strategy we have is to monitor and avoid progression from early to late AMD. Trials that have focused on the treatment of early AMD got no powerful conclusions based on previous clinical studies. Therefore, there is a pressing need to clarify the pathogenesis of AMD.

It is believed that the AMD is associated with advancing age and tobacco smoking. ${ }^{3}$ Naturally it could be conjectured that age-related structural changes in the retina are involved in the pathogenesis of AMD. Retinal pigment epithelial (RPE) cells are located in the outer layer of the retina ${ }^{4}$ and are involved in the regeneration and repair of photoreceptor cells. ${ }^{5}$ The normal RPE cells have special features. Further, loss of RPE cells could cause retinal degeneration and even blindness. It is also recognized that degenerative and progressive conditions of the RPE cells are key pathogenetic mechanisms in AMD. Besides the RPE modification was one of the most common markers for the diagnosis of AMD in clinical. ${ }^{6}$ Thus, protection 
of RPE cells from age-related changes might be important in avoiding progression from early AMD to the neovascular stage. Oxidative stress has been reported to have a key role in the aging of retinal tissues, and to have a significant effect on different ocular diseases. In general, oxidative stress is a reactive or pathological process involving accumulation of reactive oxygen species (ROS) or decreased antioxidant ability. ROS include superoxide anions $\left(\mathrm{O}^{2-}\right)$, hydroxyl radicals $(-\mathrm{OH}-)$, and hydrogen peroxide $\left(\mathrm{H}_{2} \mathrm{O}_{2}\right)$. Uncontrolled production of ROS can cause severe cellular injury. Oxidative stress would lead to less cell repair and regeneration with increasing age because of accumulation of abnormal proteins and lipids. An in vitro study in RPE cells reported that oxidative stress induced by $\mathrm{H}_{2} \mathrm{O}_{2}$ might occur as a result of reduction of the mitochondrial membrane potential, induction of cytochrome $\mathrm{c}$, and activation of caspase-3. It has also been reported that several functional genes in RPE cells in oxidative stress were expressed differently. Thus, interventions targeted to oxidation in RPE cells might be a possible treatment for early AMD.

Increasing evidence suggests that naturally occurring antioxidant agents can ameliorate age-related changes associated with oxidative damage in RPE cells. For instance, epigallocatechin-3-gallate extracted from green tea (Camellia sinensis) is a functional polyphenol with antioxidant, anticancer, and anti-inflammatory effects. ${ }^{7}$ A study by Chan et al showed that epigallocatechin-3-gallate ameliorated intracellular generation of $\mathrm{H}_{2} \mathrm{O}_{2}$ in RPE cells irradiated by ultraviolet $\mathrm{A}$ light in a concentration-dependent manner. ${ }^{8}$ Curcumin is a compound extracted from the rhizomes of Zingiberaceae and Araceae plants (Figure 1). ${ }^{9}$ A study by Jat et al show that curcumin has strong antioxidative effects and protects against lipid peroxidation, protein carbonylation, and mitochondrial permeability transition. ${ }^{10}$ However, the antioxidative effects of curcumin on the aging RPE cells are still unclear. Therefore, we conducted this in vitro study to investigate the effects of curcumin on aging RPE cells and to identify in detail the mechanisms involved in this effect.

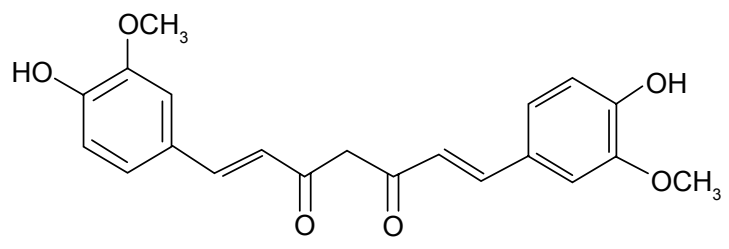

Figure I Chemical structural formula of curcumin.

\section{Materials and methods Materials}

Curcumin was obtained from Sigma Chemical Co (St Louis, MO, USA). Dulbecco's Modified Eagle Medium/ Ham's F-12 (1:1), trypsin-ethylenediaminetetraacetic acid solution, penicillin, streptomycin solution, and fetal bovine serum were obtained from Gibco (Carlsbad, CA, USA). Phenylmethylsulfonyl fluoride and 3-(4,5-dimethylthioazol2-yl)2,5-diphenyltetrazolium bromide (MTT) were sourced from Sigma Chemical Co. The Annexin V-fluorescein isothiocyanate apoptosis detection kit was obtained from Beyotime Biotechnology Co Ltd (Shanghai, People's Republic of China). The ROS detection kit was obtained from Wiig Lars Biological Technology Co Ltd (Beijing, People's Republic of China). Rabbit polyclonal antibody against caspase-3 was purchased from Abcam (Cambridge, MA, USA). Bcl-2 and Bax were from Santa Cruz Biotechnology (Santa Cruz, CA, USA). The glyceraldehyde-3-phosphate dehydrogenase antibody was purchased from Cell Signaling Technology (Danvers, MA, USA). Superoxide dismutase (SOD), maleic dialdehyde (MDA), and glutathione (GSH) test kits were obtained from Beyotime Biotechnology Co Ltd.

\section{Cell culture}

An RPE cell line (ARPE19) was sourced from the American Type Culture Collection (Manassas, VA, USA). Cells were cultured and passaged in Dulbecco's Modified Eagle's Medium/Ham's F-12 (1:1) supplemented with $5 \mathrm{mM}$ glucose, $10 \%$ fetal bovine serum, and $1 \%$ penicillin/streptomycin. The ARPE-19 cells were used at $80 \%$ confluence for each experiment and were passaged every 3 days after being transferred into culture medium. The ARPE-19 cells used were between passages 3 and 6 . The cell culture was done at $37^{\circ} \mathrm{C}$ in a humidified incubator with $5 \% \mathrm{CO}_{2}$.

\section{Aging cell model}

A pulsed $\mathrm{H}_{2} \mathrm{O}_{2}$ exposure aging cell model was generated according to the method described by Zhuge et al. ${ }^{11}$ In general, passaged ARPE-19 cells were transferred to a $10 \mathrm{~cm}$ culture dish and the cell density was controlled at approximately $50 \%$. On day 1 , the cultured cells were incubated with complete medium containing $800 \mu_{\mathrm{M} \mathrm{H}} \mathrm{O}_{2}$ for 2 hours at a temperature of $37^{\circ} \mathrm{C}$. The culture medium was then removed and the cells were rinsed with phosphate-buffered saline buffer three times. Further fresh medium was added for cell culture. From days 2-5, $\mathrm{H}_{2} \mathrm{O}_{2}$ exposure as the first day was conducted. On day 6 , the cell culture medium was 
replaced with complete medium. On day 8 , the processed RPE cells were digested with trypsin-ethylenediaminetetraacetic acid, passaged, and replated on $10 \mathrm{~cm}$ cell culture dishes.

\section{MTT assay}

The MTT assay was used to investigate cell viability. MTT dye was added to the 96-well plate and incubated overnight at $37^{\circ} \mathrm{C}$. After adding $150 \mu \mathrm{L}$ of dimethyl sulfoxide to each well, the 96-cell plates were thoroughly shocked for 10 minutes until the crystals dissolved fully. Absorbance was measured at a wavelength of $490 \mathrm{~nm}$ using an enzyme immunoassay analyzer.

\section{Flow cytometry}

The Annexin V-fluorescein isothiocyanate apoptosis detection kit (Beyotime) was used to measure apoptosis. The cultured ARPE-19 cells in all groups were trypsinized and collected by centrifugation. After two washes with icecold phosphate-buffered saline, the cells were treated with ice-cold $70 \%$ ethanol. The cells were then resuspended in $1 \mathrm{~mL}$ of phosphate-buffered saline and transferred to a new Eppendorf tube. Annexin-V fluorescein isothiocyanate and PI solution were added and cultured in the dark for 20 minutes. The cells were analyzed by flow cytometry. All detections were performed in triplicate.

\section{Measurement of ROS}

Intracellular ROS levels were measured using dihydroethidium (Wiig Lars Biological Technology Co Ltd) as the fluorescent probe. The cultured ARPE-19 cells were transferred onto a 12 -well plate at a density of $1 \times 10^{6}$ cells $/ \mathrm{mL}$. Dihydroethidium was added to each well in a working concentration of $50 \mu \mathrm{M}$. The treated cells were then incubated at $37^{\circ} \mathrm{C}$ for $15-30$ minutes in the dark, after which the plate was washed twice in phosphate-buffered saline times and the cells were used for advanced flow cytometry analysis.

\section{Detection of oxidative biomarkers}

Three common oxidative biomarkers, including, SOD, MDA, and GSH, were used to measure the oxidative stress levels in different groups. Aging RPE cells treated without or with low $(20 \mu \mathrm{M})$, medium $(40 \mu \mathrm{M})$, or high $(80 \mu \mathrm{M})$ level curcumin for 24 hours were collected. Oxidative biomarkers in these three treatment groups were then measured. In general, SOD, MDA, and GSH activity levels were detected using commercially available assay kits.
All procedures were conducted according to the manufacturer's protocol, and all samples were assayed in triplicate.

\section{Western blot analysis}

All protein in the cultured ARPE-19 cells was extracted using RIPA lysis buffer and then analyzed by Western blotting as described previously. ${ }^{12}$ Equal amounts of protein $(50 \mu \mathrm{g})$ were separated by $10 \%$ or $12 \%$ sodium dodecyl sulfatepolyacrylamide gel electrophoresis and transferred to polyvinylidene difluoride membranes, which were then blocked with $5 \%$ evaporated skimmed milk. The blots were then developed using SuperSignal-enhanced chemiluminescent substrate solution (Pierce Chemical Company, Rockford, IL, USA). The primary antibodies against Bc1-2, Bax, caspase-3, and glyceraldehyde-3-phosphate dehydrogenase were used in this study.

\section{Statistical analysis}

All the data are presented as the mean \pm standard deviation of three independent experiments. All statistical analyses were conducted using GraphPad Prism software. Differences were considered using the non-paired $t$-test or two-way analysis of variance test. $P<0.05$ was considered to be statistically significant.

\section{Results}

\section{Effect of curcumin on cell viability}

We conducted a series of studies to demonstrate the effect of curcumin on ARPE-19 cell viability in both the normal and senescent states. Cell viability was measured by MTT assay. In this in vitro experiment, curcumin had no significant effect on the viability of cells under normal conditions (Figure 2A). Curcumin $10 \mu \mathrm{M}, 20 \mu \mathrm{M}, 40 \mu \mathrm{M}, 60 \mu \mathrm{M}, 80 \mu \mathrm{M}$, and 100 $\mu \mathrm{M}$ had no significant protective effect when compared with the control group. However, in the aging state, curcumin significantly improved cell viability when compared with the control group. As showed in Figure 2B, the protective effect of curcumin on aging RPE cells was dose-dependent.

\section{Curcumin inhibits aging-induced apoptosis in ARPE-19 cells}

The aging ARPE-19 cells were considered to be apoptotic, and the rate of apoptosis was analyzed by flow cytometry. In the aging state, increased apoptotic rate was one of the key phenotype of aging change. When ARPE-19 cells were exposed to $800 \mu \mathrm{M} \mathrm{H}_{2} \mathrm{O}_{2}$ in the pulsed-wave mode, there was a significant increase in both early $(48.13 \pm 1.68$ 


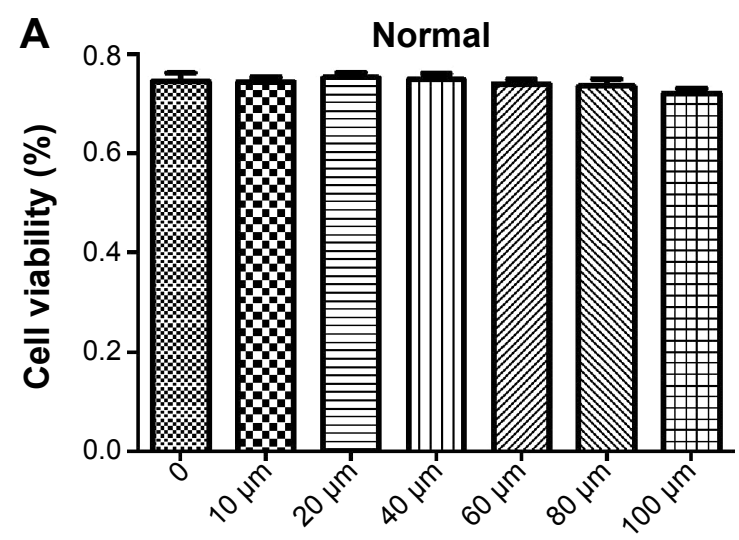

Curcumin concentrations

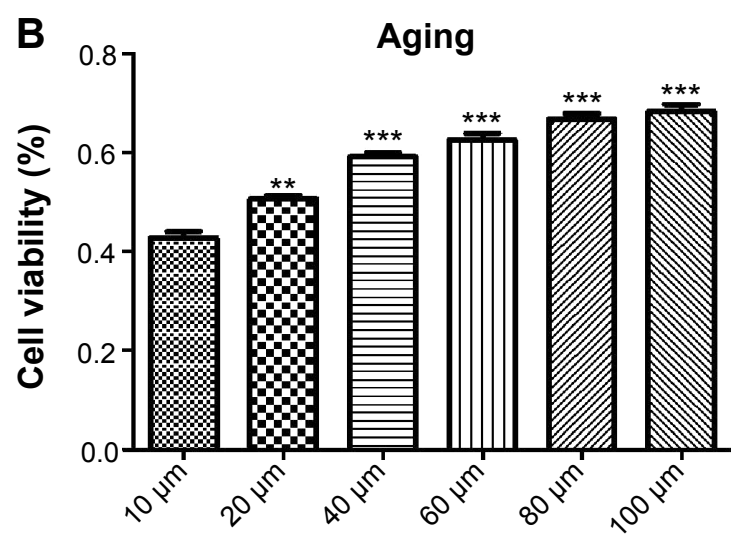

Curcumin concentrations

Figure 2 Effect of curcumin on viability of cultured ARPE- 19 cells.

Notes: (A) Effect on the normal cultured ARPE-19 cells at doses of $10 \mu \mathrm{M}, 20 \mu \mathrm{M}, 40 \mu \mathrm{M}, 60 \mu \mathrm{M}, 80 \mu \mathrm{M}$, and I00 $\mu \mathrm{M}$ and (B) effect on aging ARPE-19 cells at doses of $10 \mu \mathrm{M}, 20 \mu \mathrm{M}, 40 \mu \mathrm{M}, 60 \mu \mathrm{M}, 80 \mu \mathrm{M}$, and $100 \mu \mathrm{M}$. **P<0.0I; ***P<0.00I.

versus $3.93 \pm 0.31, P<0.001)$ and late $(1.46 \pm 0.29$ versus $0.00 \pm 0.00, P<0.001)$ apoptosis when compared with the control group. In the curcumin-treated groups, rates of apoptosis were significantly decreased by all doses given, except for late apoptosis in the $20 \mu \mathrm{M}$ curcumin group. The improvement in apoptotic state induced by curcumin was also dose-dependent. Augmentation of the curcumin dose was associated with a significant decrease in both early and late apoptotic rates. Further, rates of both early (11.83 \pm 1.13$)$ and late $(0.00 \pm 0.00)$ apoptosis were significantly decreased in the high-dose curcumin group when compared with the low-dose curcumin group $(32.37 \pm 0.75, P<0.001)$. Representative flow cytometry data and detailed results are shown in Figure 3.

\section{Effect of curcumin on apoptosis- associated proteins}

Having confirmed that curcumin could affect aging-induced apoptosis in our cell model, we went on to investigate the effects of curcumin on expression of apoptosis-associated

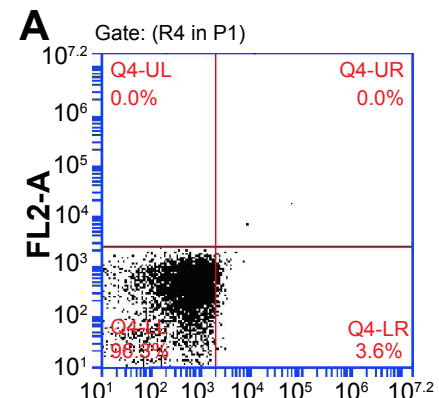

FL1-A

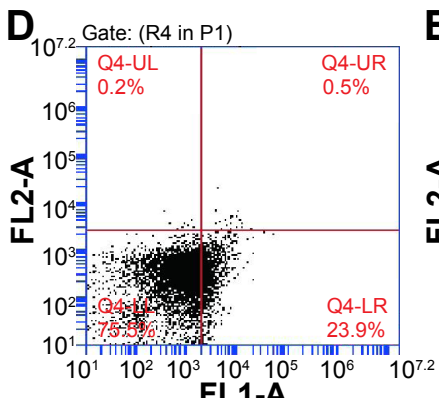

FL1-A

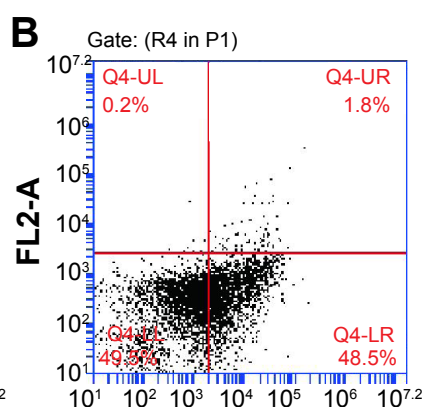

FL1-A

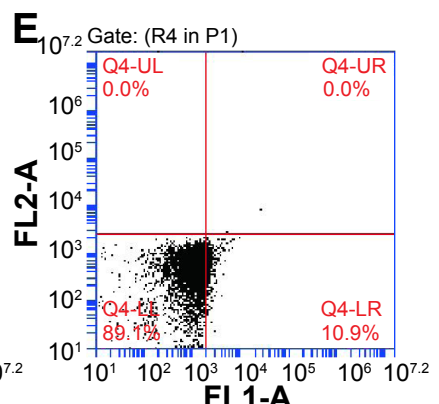

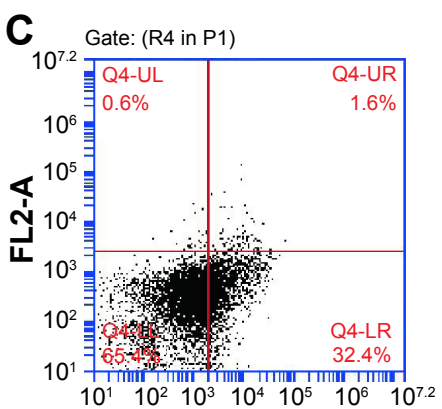

FL1-A

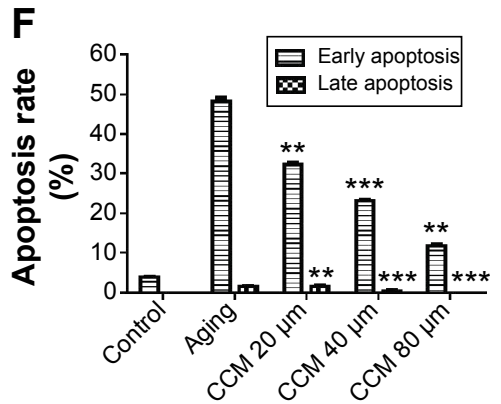

Figure 3 Effect of curcumin on apoptosis in cultured ARPE-19 cells.

Notes: (A) Normal cultured cells. (B) Aging ARPE-19 cell model. (C) Effect of $20 \mu$ curcumin on apoptosis in cultured ARPE-19 cells. (D) Effect of $40 \mu$ M curcumin on apoptosis in cultured ARPE-19 cells. (E) Effect of $80 \mu \mathrm{M}$ curcumin on apoptosis in cultured ARPE-19 cells. (F) Summary of early and late apoptosis rates in each group. Differences in comparisons of each dosage of curcumin group and aging group were presented. $* * P<0.0 \mathrm{I} ; * * * P<0.00 \mathrm{I}$.

Abbreviation: CCM, curcumin. 


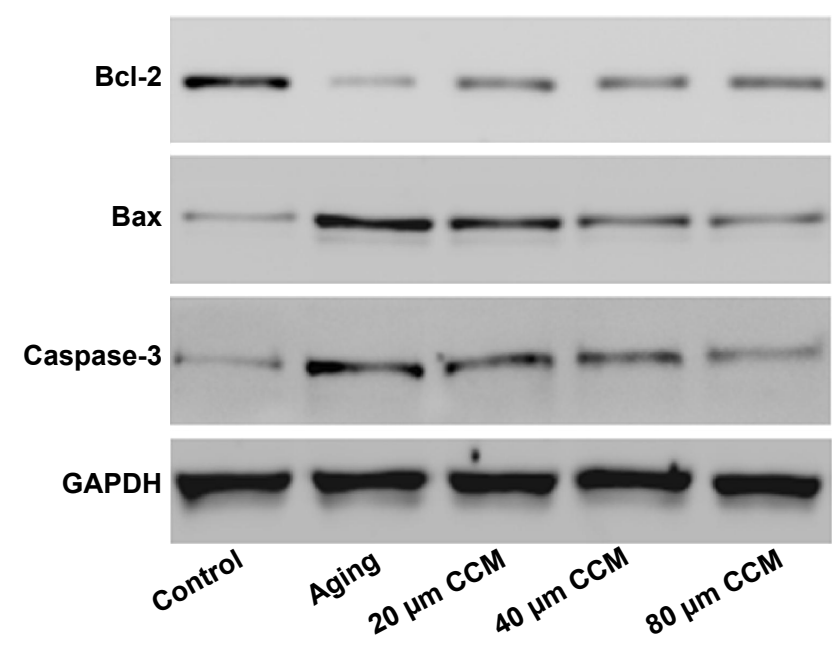

Figure 4 Role of curcumin treatment in expression of apoptosis-associated genes, including Bax, Bcl-2, and caspase-3, by Western blotting.

Note: When compared with the aging group, Bax and caspase- 3 treated by curcumin were downregulated while $\mathrm{Bcl}-2$ was upregulated in dose-response manner.

Abbreviations: CCM, curcumin; GAPDH, glyceraldehyde-3-phosphate dehydrogenase.

genes, ie, Bax, Bcl-2, and caspase-3, by Western blotting. Compared with normally cultured ARPE-19 cells, the aging cells contained lower levels of Bcl-2 and higher levels of Bax and caspase-3, which was consistent with our flow cytometry results and with the fact that Bcl-2 is anti-apoptotic and Bax and caspase- 3 are apoptotic. We used curcumin at three different concentrations to detect their effects on these apoptosis-associated proteins. When compared with the aging group, the expression of Bax and caspase- 3 were decreased when treated by curcumin. Further, levels of Bcl-2, an anti-apoptotic protein, were upregulated when the concentration of curcumin was increased. When the effects of the different concentrations of curcumin were detected, the trend increased of Bcl-2 and decreased of Bax and caspase- 3 were detected. When oxidative related proteins treated with different curcumin concentrations also presented the same trend. In general, there was no difference in Bcl-2 and caspase-3 levels between the curcumin $20 \mu \mathrm{M}$ and $40 \mu \mathrm{M}$ groups. However, downregulation of Bcl-2 in the curcumin $80 \mu \mathrm{M}$ group was not significant compared with that in the $20 \mu \mathrm{M}$ and $40 \mu \mathrm{M}$ groups. While the tendency of each protein expression in increasing curcumin treated groups was significant. Detailed results are shown in Figure 4.

\section{Curcumin reduces intracellular ROS content in aging ARPE-I 9 cells}

In general, intracellular ROS concentrations were significantly increased in aging ARPE-19 cells as detected by the specific fluorescent probe used in our study. Compared with normally cultured RPE cells, the aging RPE cells contained higher intracellular ROS levels $(6,064.66 \pm 73.98$ versus $2,585.24 \pm 69.39, P<0.001)$. Intracellular ROS concentrations after treatment with low, middle, and high concentrations of curcumin were 4,919.68 $\pm 60.38,3,988.97 \pm 48.31$, and 3,215.40 \pm 55.91 , respectively (Figure 5). With increasing concentrations of curcumin, the ROS content decreased accordingly. These findings indicate that treatment with curcumin $20 \mu \mathrm{M}, 40 \mu \mathrm{M}$, and $80 \mu \mathrm{M}$ can effectively prevent age-related production of ROS. Detailed figures and data can be seen in Figure 5.

\section{Effect of curcumin on oxidative stress biomarkers in aging RPE cells}

Given that oxidative stress is quite important in the aging status of RPE cells, we measured the levels of three oxidative stress biomarkers, ie, MDA, SOD, and GSH, in cultured ARPE-19 cells in the different treatment groups. Expression of MDA was higher in the aging group, while SOD and GSH expression was lower $(P<0.001)$. In addition, levels of the anti-apoptotic proteins, SOD and GSH, were significantly higher in the aging ARPE-19 cells treated with curcumin than in the non-treated group. However, the MDA level was significantly lower after incubation with curcumin in the aging RPE cells $(P<0.001)$. The MDA level was downregulated with the increasing concentrations of curcumin used in this study, and SOD and GSH were upregulated accordingly. The results for these oxidative stress biomarkers in aging RPE cells with and without curcumin treatment are presented in Figure 6.

\section{Discussion}

AMD is now one of the most important causes of blindness worldwide, so confirmation of a protective effect of curcumin would advance development of potential therapeutic measures. RPE cells lie behind the photoreceptor cells and are susceptible to oxidative stress. Several important antioxidative effects of curcumin on the RPE cells were detected in the present study. Firstly, we identified lower cell viability, higher apoptosis rates, and more severe oxidative status in the aging RPE cell model when compared with normal cells. Secondly, curcumin improved cell viability, apoptotic rates, and oxidative stress levels in aging ARPE-19 cells. Finally, curcumin significantly influenced the expression of apoptosisassociated proteins and oxidative stress biomarkers.

Considering that older age is a definitive risk factor for AMD, a protective intervention for retinal aging would 

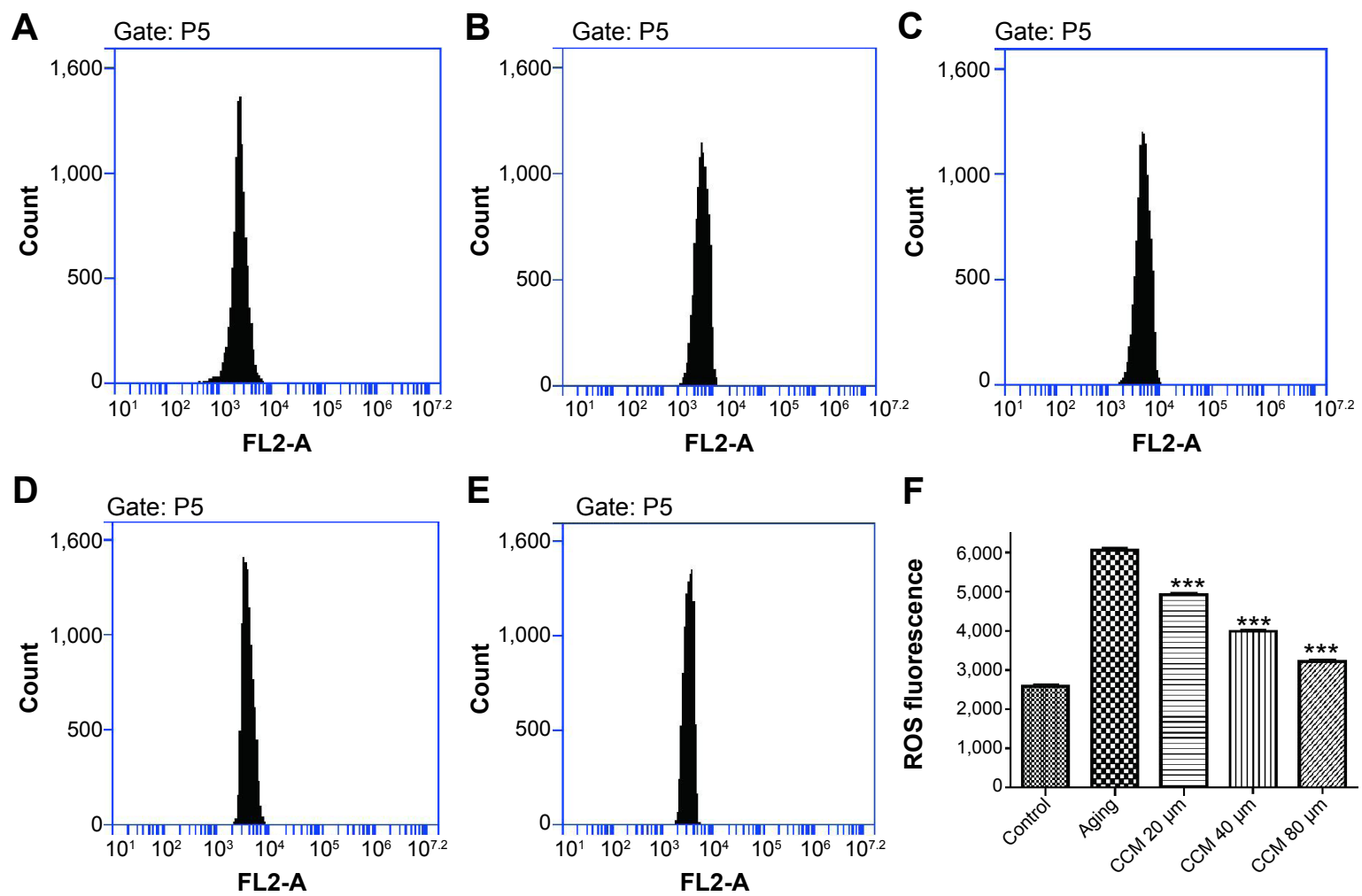

Figure 5 Effect of curcumin on ROS content in cultured ARPE-19 cells.

Notes: (A) Normal cultured cells used as the control. (B) Aging ARPE-19 cell model used as the positive control. (C) Effect of $20 \mu \mathrm{M}$ curcumin on ROS content in cultured ARPE- 19 cells. (D) Effect of $40 \mu$ M curcumin on ROS content in cultured ARPE-1 9 cells. (E) Effect of $80 \mu M$ curcumin on ROS content in cultured ARPE-19 cells. (F) Summary of ROS content in each group. All studies were conducted in triplicate. $* * * P<0.001$.

Abbreviations: CCM, curcumin; ROS, reactive oxygen species.

provide better therapeutic measures for AMD. ${ }^{13}$ Curcumin has been reported to have a protective effect in a number of diseases, including hyperglycemia, ${ }^{14}$ carcinoma, ${ }^{15}$ and hypertension. ${ }^{16}$ Further Deng et al have reported that curcumin is a potential therapy for Alzheimer's disease (AD). ${ }^{17}$ In general, the protective effect of curcumin in $\mathrm{AD}$ was related to its anti-inflammatory and antioxidative effects. ${ }^{18}$ AMD and $\mathrm{AD}$ are both age-related diseases of the human central nervous system, ${ }^{19}$ and share several common pathogenic mechanisms. ${ }^{20}$ Accordingly, treatment for AD might also be effective for AMD. Given that a number of previous studies have investigated the effects of curcumin in $\mathrm{AD}$, it is logical to investigate its potential protective effects in AMD.

There have already been several reports of the use of curcumin in retinal disorders. Zuo et al reported that curcumin had protective effects on retinal Müller cells in early diabetic rats. ${ }^{21}$ Compared with controls, MDA was upregulated in the retinal tissues of these diabetic rats. Another study showed that curcumin could suppress N-methyl-N-nitrosourea (MNU)induced photoreceptor apoptosis in an animal model. ${ }^{22}$ Comparing photoreceptor apoptosis in Sprague-Dawley rats injected with MNU + curcumin or MNU only, it was found that curcumin $200 \mathrm{mg} / \mathrm{kg}$ significantly suppressed the loss of photoreceptor cells. It was concluded that curcumin could inhibit MNU-induced photoreceptor cell apoptosis by suppressing DNA oxidative stress.

As RPE cell layer was the most common oxidativedamaged structure in the retina, the effect of antioxidative curcumin in RPE cells was also studied. A study by Chang et al showed that curcumin significantly decreased ROS in AMD-RPEs. ${ }^{23}$ Advanced in vitro study showed that curcumin activated a number of oxidative stress-regulated genes, including those for platelet-derived growth factor, vascular endothelial growth factor, insulin-like growth factor-binding protein 2, and SOD2. Accordingly, our present data indicate that curcumin might be an effective treatment option for AMD because of its ability to decrease aging-associated oxidative stress. In 2013, Li et al conducted an in vitro study to assess the effect on a curcumin analog on acrolein-induced toxicity in ARPE-19 cells. ${ }^{24}$ Curcumin at a concentration of $5 \mu \mathrm{M}$ could completely protect RPE cells from acroleininduced oxidative cell damage and restore GSH levels and mitochondrial function. A high-throughput study by Howell et al investigated the effect of curcumin on expression of 

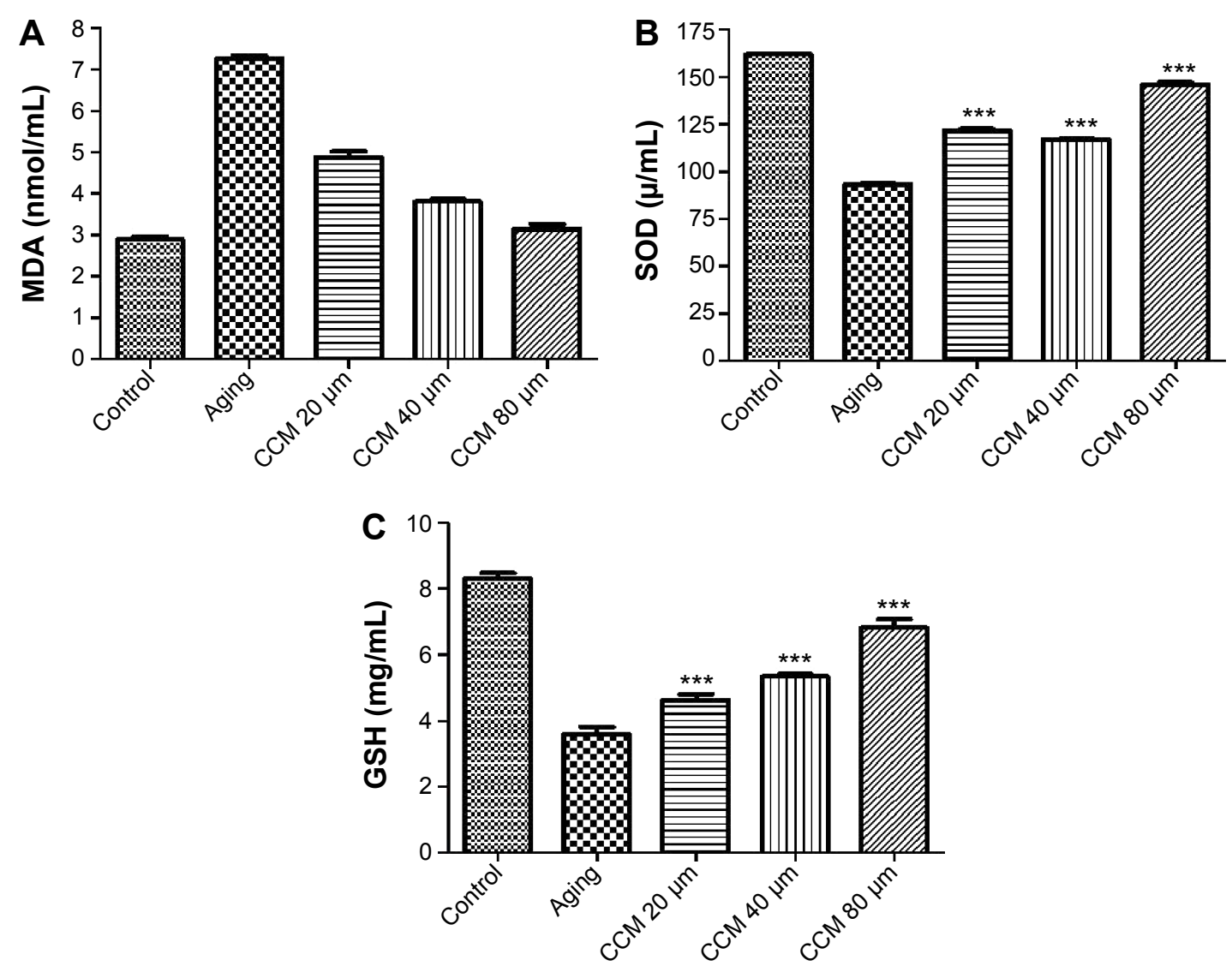

Figure 6 Effect of curcumin on oxidative biomarkers in cultured ARPE-19 cells.

Notes: (A) Expression of MDA in control, aging, and $20 \mu \mathrm{M}, 40 \mu \mathrm{M}$, and $80 \mu \mathrm{M}$ curcumin groups. (B) Expression of SOD in control, aging, and $20 \mu \mathrm{M}, 40 \mu \mathrm{M}$, and $80 \mu \mathrm{M}$ curcumin groups. (C) Expression of GSH in control, aging, and $20 \mu \mathrm{M}, 40 \mu \mathrm{M}$, and $80 \mu \mathrm{M}$ curcumin group. $* * * P<0.00 \mathrm{I}$.

Abbreviations: CCM, curcumin; GSH, glutathione; MDA, maleic dialdehyde; SOD, superoxide dismutas.

miRNAs in ARPE-19 cells. ${ }^{25}$ In that study, 18 miRNAs were downregulated and 29 miRNAs were upregulated. Besides, incubation with curcumin significantly decreased $\mathrm{H}_{2} \mathrm{O}_{2}$-induced expression of 17 miRNAs. Thus, it could be presumed that miRNAs are important effector molecules when curcumin is used in the treatment of AMD.

In this study, we adopted a pulsed $\mathrm{H}_{2} \mathrm{O}_{2}$ exposure model as in vitro aging model. This cellular model produced more significant feasibility. Compared with one-time $\mathrm{H}_{2} \mathrm{O}_{2}$ exposure, this in vitro model would provide more accurate results. In this study we focused on the protectiveness of curcumin on oxidative stress and apoptosis in the aging RPE cells. We also identified both biological changes in cells and differently expressed oxidative related proteins in the curcumin treated group. The positive and interactive results in this study provide useful evidence for application of curcumin in the treatment of AMD. However, ours was an in vitro study, and its conclusions need validating by well-designed in vivo studies.

In conclusion, curcumin was able to regulate proliferation, oxidative stress, and apoptosis in aging RPE cells.
Our findings, together with those of previous studies, suggest a potentially important role for curcumin on the protection of RPE cells during development of AMD. Accordingly, application of curcumin may be a novel strategy to protect against aging change in the AMD.

\section{Disclosure}

The authors report no conflicts of interest in this work.

\section{References}

1. Vassilev ZP, Ruigomez A, Soriano-Gabarro M, Garcia Rodriguez LA. Diabetes, cardiovascular morbidity, and risk of age-related macular degeneration in a primary care population. Invest Ophthalmol Vis Sci. 2015;56: $1585-1592$.

2. Wolf S. Current status of anti-vascular endothelial growth factor therapy in Europe. Jpn J Ophthalmol. 2008;52:433-439.

3. Zanon-Moreno V, Garcia-Medina JJ,Zanon-Viguer V, Moreno-Nadal MA, Pinazo-Duran MD. Smoking, an additional risk factor in elder women with primary open-angle glaucoma. Mol Vis. 2009;15: 2953-2959.

4. Zhang X, Hughes BA. KCNQ and KCNE potassium channel subunit expression in bovine retinal pigment epithelium. Exp Eye Res. 2013; 116:424-432. 
5. Majji AB, de Juan E, Jr. Retinal pigment epithelial autotransplantation: morphological changes in retina and choroid. Graefes Arch Clin Exp Ophthalmol. 2000;238:779-791.

6. Subhi Y, Henningsen GO, Larsen CT, Sorensen MS, Sorensen TL. Foveal morphology affects self-perceived visual function and treatment response in neovascular age-related macular degeneration: a cohort study. PLoS One. 2014;9:e91227.

7. Thichanpiang $\mathrm{P}$, Wongprasert K. Green tea polyphenol epigallocatechin-3-gallate attenuates TNF-alpha-induced intercellular adhesion molecule-1 expression and monocyte adhesion to retinal pigment epithelial cells. Am J Chin Med. 2015;43:103-119.

8. Chan CM, Huang JH, Lin HH, et al. Protective effects of (-)-epigallocatechin gallate on UVA-induced damage in ARPE19 cells. Mol Vis. 2008; 14:2528-2534.

9. Zhao WC, Zhang B, Liao MJ, et al. Curcumin ameliorated diabetic neuropathy partially by inhibition of NADPH oxidase mediating oxidative stress in the spinal cord. Neurosci Lett. 2014;560:81-85.

10. Jat D, Parihar P, Kothari SC, Parihar MS. Curcumin reduces oxidative damage by increasing reduced glutathione and preventing membrane permeability transition in isolated brain mitochondria. Cell $\mathrm{Mol} \mathrm{Biol}$ (Noisy-le-grand). 2013;59 Suppl:OL1899-OL1905.

11. Zhuge CC, Xu JY, Zhang J, et al. Fullerenol protects retinal pigment epithelial cells from oxidative stress-induced premature senescence via activating SIRT1. Invest Ophthalmol Vis Sci. 2014;55:4628-4638.

12. Liu B, Li S, Su Y, Xiong M, Xu Y. Comparative study of the protective effects of terfenadine and amiodarone on barium chloride/aconitineinduced ventricular arrhythmias in rats: a potential role of terfenadine. Mol Med Rep. 2014;10:3217-3226.

13. Blagosklonny MV. Validation of anti-aging drugs by treating age-related diseases. Aging (Albany NY). 2009;1:281-288.

14. Ghorbani Z, Hekmatdoost A, Mirmiran P. Anti-hyperglycemic and insulin sensitizer effects of turmeric and its principle constituent curcumin. Int J Endocrinol Metab. 2014;12:e18081.

15. Rana C, Piplani H, Vaish V, Nehru B, Sanyal SN. Downregulation of telomerase activity by diclofenac and curcumin is associated with cell cycle arrest and induction of apoptosis in colon cancer. Tumour Biol. March 6, 2015. [Epub ahead of print].
16. Sangartit W, Kukongviriyapan U, Donpunha W, et al.Tetrahydrocurcumin protects against cadmium-induced hypertension, raised arterial stiffness and vascular remodeling in mice. PLoS One. 2014;9:e114908.

17. Deng Y, Lu X, Wang L, et al. Curcumin inhibits the AKT/NF-kappaB signaling via $\mathrm{CpG}$ demethylation of the promoter and restoration of NEP in the N2a cell line. AAPS J. 2014;16:649-657.

18. McClure R, Yanagisawa D, Stec D, et al. Inhalable curcumin: offering the potential for translation to imaging and treatment of Alzheimer's disease. J Alzheimers Dis. 2015;44:283-295.

19. Zhao Y, Bhattacharjee S, Jones BM, et al. Beta-amyloid precursor protein (betaAPP) processing in Alzheimer's disease (AD) and age-related macular degeneration (AMD). Mol Neurobiol. September 10, 2014. [Epub ahead of print].

20. Parsons CG, Ruitenberg M, Freitag CE, et al. MRZ-99030 - a novel modulator of Abeta aggregation: I Mechanism of action (MoA) underlying the potential neuroprotective treatment of Alzheimer's disease, glaucoma and age-related macular degeneration (AMD). Neuropharmacology. 2015;92:158-169.

21. Zuo ZF, Zhang Q, Liu XZ. Protective effects of curcumin on retinal Muller cell in early diabetic rats. Int J Ophthalmol. 2013;6:422-424.

22. Emoto Y, Yoshizawa K, Uehara N, et al. Curcumin suppresses N-methyl$\mathrm{N}$-nitrosourea-induced photoreceptor apoptosis in Sprague-Dawley rats. In Vivo. 2013;27:583-590.

23. Chang YC, Chang WC, Hung KH, et al. The generation of induced pluripotent stem cells for macular degeneration as a drug screening platform: identification of curcumin as a protective agent for retinal pigment epithelial cells against oxidative stress. Front Aging Neurosci. 2014;6:191.

24. LiY,ZouX,CaoK, etal. Curcuminanalog 1,5-bis(2-trifluoromethylphenyl)-1, 4-pentadien-3-one exhibits enhanced ability on Nrf2 activation and protection against acrolein-induced ARPE-19 cell toxicity. Toxicol Appl Pharmacol. 2013;272:726-735.

25. Howell JC, Chun E, Farrell AN, et al. Global microRNA expression profiling: curcumin (diferuloylmethane) alters oxidative stress-responsive microRNAs in human ARPE-19 cells. Mol Vis. 2013;19:544-560.
Drug Design, Development and Therapy

\section{Publish your work in this journal}

Drug Design, Development and Therapy is an international, peerreviewed open-access journal that spans the spectrum of drug design and development through to clinical applications. Clinical outcomes, patient safety, and programs for the development and effective, safe, and sustained use of medicines are a feature of the journal, which

\section{Dovepress}

has also been accepted for indexing on PubMed Central. The manuscript management system is completely online and includes a very quick and fair peer-review system, which is all easy to use. Visit http://www.dovepress.com/testimonials.php to read real quotes from published authors. 